\title{
Cosmic-ray interactions with the Sun using the FLUKA code
}

\author{
M. N. Mazziotta $\odot,{ }^{1, *}$ P. De La Torre Luque, ${ }^{1,2}$ L. Di Venere $\odot,{ }^{3}$ A. Fassò, ${ }^{4}$ A. Ferrari, ${ }^{5}$ \\ F. Loparco $\oplus^{1,2}$ P. R. Sala, ${ }^{6}$ and D. Serini ${ }^{1,2}$ \\ ${ }^{1}$ Istituto Nazionale di Fisica Nucleare, Sezione di Bari, via Orabona 4, I-70126 Bari, Italy \\ ${ }^{2}$ Dipartimento di Fisica "M. Merlin” dell'Università e del Politecnico di Bari, \\ via Amendola 173, I-70126 Bari, Italy \\ ${ }^{3}$ Istituto Nazionale di Fisica Nucleare, Sezione di Bari, I-70126 Bari, Italy \\ ${ }^{4} 13$ Passage Hamo, CH-1262 EYSINS, Switzerland \\ ${ }^{5}$ CERN, the European Organization for Nuclear Research, \\ Esplanade des Particules 1, 1211 Geneva, Switzerland \\ ${ }^{6}$ Istituto Nazionale di Fisica Nucleare, Sezione di Milano, Via Celoria,16, 20133 Milano, Italy
}

(Received 29 January 2020; accepted 2 March 2020; published 8 April 2020; corrected 27 October 2020)

The interactions of cosmic rays with the solar atmosphere produce secondary particles which can reach the Earth. In this work, we present a comprehensive calculation of the yields of secondary particles such as gamma-rays, electrons, positrons, neutrons, and neutrinos performed with the FLUKA code. We also estimate the intensity at the Sun and the fluxes at the Earth of these secondary particles by folding their yields with the intensities of cosmic rays impinging on the solar surface. The results are sensitive to the assumptions on the magnetic field nearby the Sun and to the cosmic-ray transport in the magnetic field in the inner Solar System.

DOI: 10.1103/PhysRevD.101.083011

\section{INTRODUCTION}

Cosmic rays entering in the Solar System after propagating for millions of years in the Galaxy can reach the planets and the Sun itself, producing emission of secondary particles, such as gamma rays and neutrinos, due to the interactions with the surfaces or the atmospheres of the celestial bodies.

The Moon [1,2] and the Earth [3] are both bright sources of gamma rays. Lunar and terrestrial gamma rays are originated from the hadronic interactions of cosmic-ray nuclei with the lunar surface and with the upper layers of the Earth's atmosphere, respectively. The Sun is also a bright source of high-energy gamma rays. While gamma rays from the Earth and from the Moon are originated from cosmic-ray nuclei, the solar gamma-ray emission consists of two components: the first one, called disk emission, is due to cosmic-ray nuclei interacting with the solar surface $[4,5]$ and is localized around the solar disk; the second one, which is due to the inverse Compton scatterings of cosmicray electrons (and positrons) with the solar optical photons,

\footnotetext{
mazziotta@ba.infn.it;
}

Published by the American Physical Society under the terms of the Creative Commons Attribution 4.0 International license. Further distribution of this work must maintain attribution to the author(s) and the published article's title, journal citation, and DOI. is a diffuse component and extends up to tens of degrees from the Sun $[4,6-8]$.

Several attempts have already been made to calculate the secondary emission (e.g., gamma rays and neutrinos) due to the interactions of cosmic rays with the solar atmosphere (see, for example, Refs. [5,9]). In particular, the knowledge of such emission could be used to constrain exotic processes, such as the production of standard model particles in the annihilation of dark matter particles captured by the Sun [9-14].

Early predictions are based on semianalytical calculations with the inclusion of solar magnetic field [5], while full numerical simulations for the production of neutrinos based on the Monte Carlo method have been performed in Ref. [15] and recently revisited and updated by Refs. [9]. However, in those Monte Carlo simulations, the effect of the magnetic field was neglected since the calculation was performed at high energies. The production of neutrinos is closely related to that of gamma rays in the solar disk, as both are originated from hadronic interactions of cosmic-ray nuclei.

In this work, we have performed a full simulation with the FLUKA code to calculate the yields of secondary particles produced by the interactions of cosmic rays with the Sun. In particular, we have simulated the interactions of protons, helium nuclei, and electrons impinging on the solar atmosphere in a wide range of kinetic energy per nucleon from $0.1 \mathrm{GeV} / \mathrm{n}$ to $100 \mathrm{TeV} / \mathrm{n}$, while the energy of secondary particles has been simulated down to $100 \mathrm{keV}$. The low-energy region is extremely interesting for the 
proposed future gamma-ray telescopes [16-18], which aim to probe photon energy intervals extending well below the lower bound of that explored by the Fermi Large Area Telescope (a few tens of MeV) [19].

The present work is based on our previous ones, in which we evaluated, using FLUKA, the yields of secondary cosmic rays in the collisions of primary cosmic rays with the interstellar gas [20] and the lunar gamma-ray emission [2].

In simulating the interactions of cosmic rays with the solar atmosphere, there are a number of important effects to consider. First, the interplanetary magnetic field affects the spectra of cosmic rays reaching the Sun. Second, the strong heliospheric magnetic field nearby the Sun also affects the trajectories of charged particles in the solar atmosphere: in particular, the total path length increases with the intensity of the magnetic field, and this corresponds to an increase of the interaction probability and consequently to an increase of the cascades of secondary particles. Finally, the profile of the solar atmosphere needs to be accounted in detail, since the cascades usually develop from a low-density medium toward a denser medium; in addition, the yield of secondary particles far away from the Sun is also affected by the grammage along the line of the sight from the production point to the outer space.

\section{SIMULATION SETUP}

The propagation and the interactions of cosmic rays with the solar atmosphere have been simulated with the FLUKA code [21-23]. FLUKA is a general-purpose Monte Carlo code for the simulation of hadronic and electromagnetic interactions, used in many applications. It can simulate with high accuracy the interactions and propagation in matter of about 60 different species of particles, including photons and electrons from $1 \mathrm{keV}$ to thousands of $\mathrm{TeV}$, neutrinos, muons of any energy, hadrons of energies up to $20 \mathrm{TeV}$ (up to $10 \mathrm{PeV}$ when it is interfaced with the DPMJET code [24]) and all their antiparticles, neutrons down to thermal energies, and heavy ions.

Hadronic interactions are treated in FLUKA following a theory-driven approach. Below a few GeV, the hadronnucleon interaction model is based on resonance production and decay of particles, while for higher energies, the Dual Parton Model is used, implying a treatment in terms of quark chain formation and hadronization. The extension from hadron-nucleon to hadron-nucleus interactions is done in the framework of the Preequilibrium Approach to Nuclear Thermalization model (PEANUT) [25,26], including the Gribov-Glauber multicollision mechanism followed by the preequilibrium stage and eventually equilibrium processes (evaporation, fission, Fermi breakup, and gamma deexcitation).
FLUKA can handle even very complex geometries, using an improved version of the well-known Combinatorial Geometry package, which has been designed to track correctly both neutral and charged particles, even in the presence of magnetic fields.

In our code, we use a spherical reference frame centered on the Sun, which is described as a sphere of radius $R_{\odot}=6.9551 \times 10^{10} \mathrm{~cm}$. The polar axis (i.e., $\mathrm{z}$ axis) of the reference frame corresponds to the Sun's rotation axis. Our simulation includes the radial profiles of the chemical composition, of the density, of the temperature, and of the pressure of the Sun (see Sec. II A). In addition, we have implemented various models of the magnetic field in the region close to the Sun (inner magnetic field; see Sec. II B), while for the interplanetary magnetic field, we have used the Parker model (see Sec. II C). As will be discussed in the next sections, the inner magnetic field affects the cosmicray interactions with the solar environment, while the interplanetary magnetic field affects their propagation to the Sun.

To evaluate the yields of secondary particles from the Sun, we have simulated several samples of protons, electrons, and ${ }^{4} \mathrm{He}$ nuclei with different kinetic energies impinging a sphere of radius $R_{S S}=2.5 R_{\odot}$ surrounding the Sun, with an isotropic and uniform distribution. As it will be shown in Secs. II B and II C, the generation sphere corresponds to the boundary between the inner and outer magnetic field regions. The primary kinetic energy values are taken on a grid of 97 equally spaced values in a logarithmic scale, from $100 \mathrm{MeV} / \mathrm{n}$ up to $100 \mathrm{TeV} / \mathrm{n}$.

\section{A. Solar composition}

In our simulation, we have implemented a chemical composition profile of the Sun derived from the standard solar models (SSMs) for the interior of the Sun, provided by Ref. [27] (hereafter model gs98). ${ }^{1}$ Figure 1 shows the mass fractions of the main components as a function of the distance from the center of the Sun for the gs 98 model. The main components are the hydrogen and ${ }^{4} \mathrm{He}$, while the abundances of heavier isotopes are below 1\%. Since most of cosmic-ray interactions will take place in the solar atmosphere, close to the surface of the Sun, we have extrapolated this model outside the Sun, assuming that the chemical composition of the atmosphere is the same as that at $r=R_{\odot}$.

For the radial profiles of density, temperature, and pressure, we use the model provided by Ref. [28] (hereafter model S), since it extends up to about $500 \mathrm{~km}$ above the solar surface. We then extrapolate this model to higher altitudes, up to about $1400 \mathrm{~km}$. We have also verified that model gs98 is very similar to model $\mathrm{S}$ up to $r=R_{\odot}$.

\footnotetext{
${ }^{1}$ We use the data file http://www.ice.csic.es/personal/aldos/ Solar_Data_files/struct_b16_gs98.dat.
} 


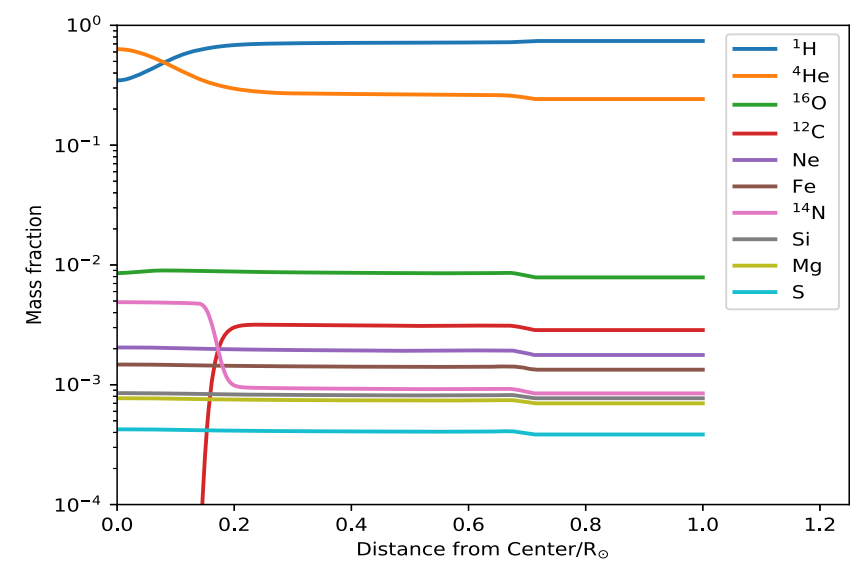

FIG. 1. Mass fractions in the gs98 model as a function of the distance from the center of the Sun in units of $R_{\odot}$. Only atoms with mass fractions above $10^{-4}$ at the surface are shown. The data are taken from Ref. [27].

Figure 2 shows the radial density (top panel), pressure (middle panel), and temperature (bottom panel) profiles. Model S is shown with black points, the model gs98 is shown with blue lines, and the extrapolation is shown with red lines.

In the FLUKA simulation setup, we have implemented 100 layers (i.e., shells) with different densities and chemical compositions, divided in three sets equally spaced on a logarithmic density scale: the external 40 layers from about $10^{-13}$ up to $10^{-3} \mathrm{~g} / \mathrm{cm}^{3}$, the middle 40 layers from $10^{-3}$ to $10^{-1} \mathrm{~g} / \mathrm{cm}^{3}$, and the inner 20 layers with density greater than $10^{-1} \mathrm{~g} / \mathrm{cm}^{3}$. In each shell, we define a compound mixture material according to the mass composition, density, temperature, and pressure profiles shown in Figs. 1 and 2. We have also implemented the temperature profile, since the neutron cross sections for the main isotopes (i.e., $\mathrm{H},{ }^{3} \mathrm{He},{ }^{4} \mathrm{He}$, and ${ }^{12} \mathrm{C}$ ) are dependent on the temperature. In particular, the temperature has an effect in the capture of neutrons that produce the gamma-ray line of $2.2 \mathrm{MeV}$.

\section{B. Inner magnetic field}

The magnetic field near the Sun is complex and strongly time dependent, and the coronal magnetic field is usually extrapolated from the observed photospheric fields. A widely adopted model is the potential field source surface (PFSS) model $[29,30]$, in which the field is purely radial on a sphere of radius $R_{S S}$ (source surface). In our simulation, we have implemented the field maps taken from the Solar Dynamics Observatory Joint Science Operations Center (JSOC) [31,32], which are calculated starting from the photospheric magnetic field observations [33-35] of the Helioseismic and Magnetic Imager (HMI) [36], the Solar Dynamics Observatory [37], and the Michelson Doppler Imager [38] instrument on the Solar and Heliospheric Observatory [39] and assuming $R_{S S}=2.5 R_{\odot}$. In each
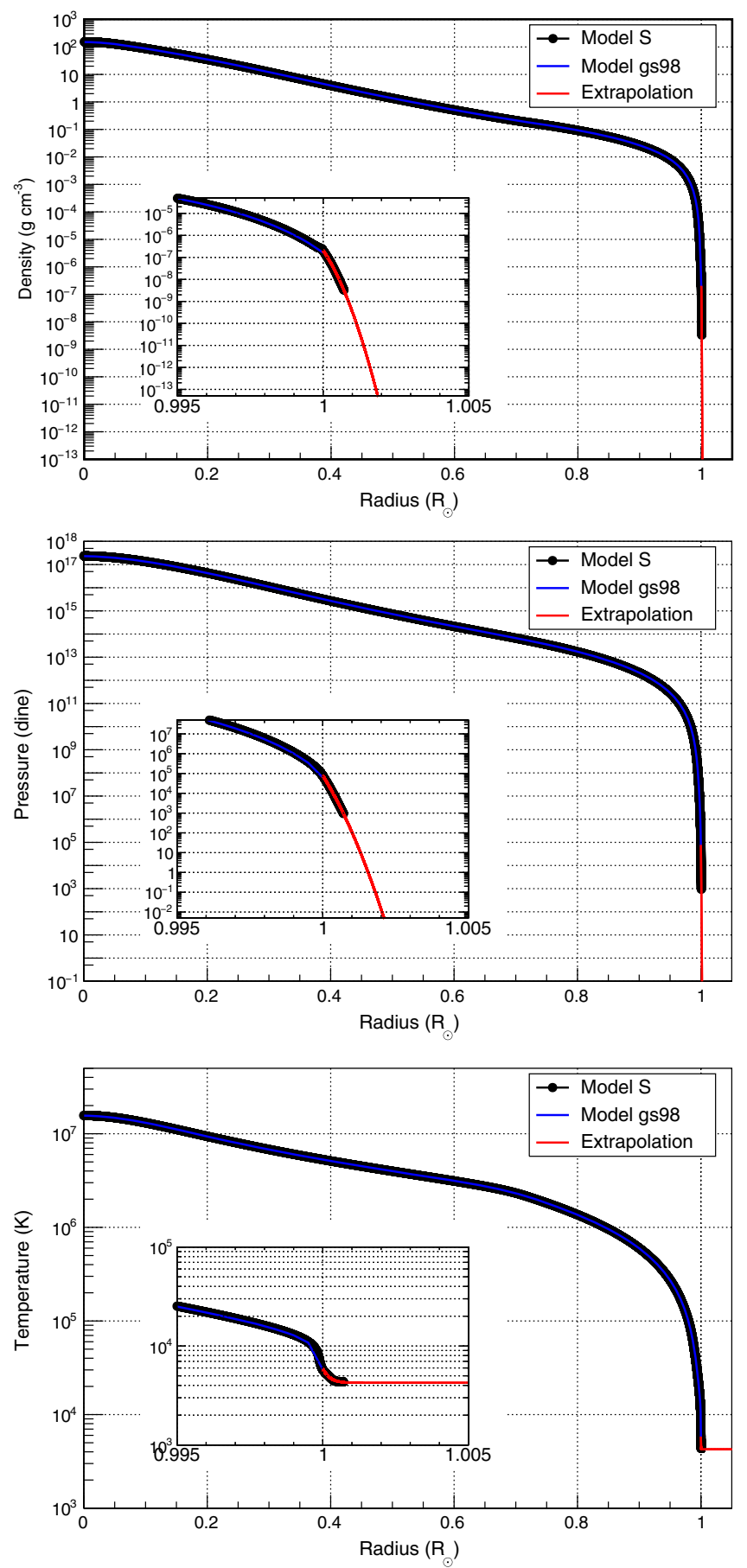

FIG. 2. Density (top panel), pressure (middle panel), and temperature (bottom panel) as a function of the radial distance from the Sun center in units of $R_{\odot}$. The black dots indicate the model S [28]; the blue line is the model gs98 [27]; the red line is the present extrapolation. The inset shows a zoom near the Sun radius.

map, the three components of the coronal magnetic field, $\left(B_{r}, B_{\theta}, B_{\phi}\right)$ are tabulated at 51 heights between the photosphere $\left(r=R_{\odot}\right)$ and the source surface $\left(r=R_{S S}\right)$. The field maps are available starting from the Carrington Rotation (CR) 2097 (May-June 2010). 


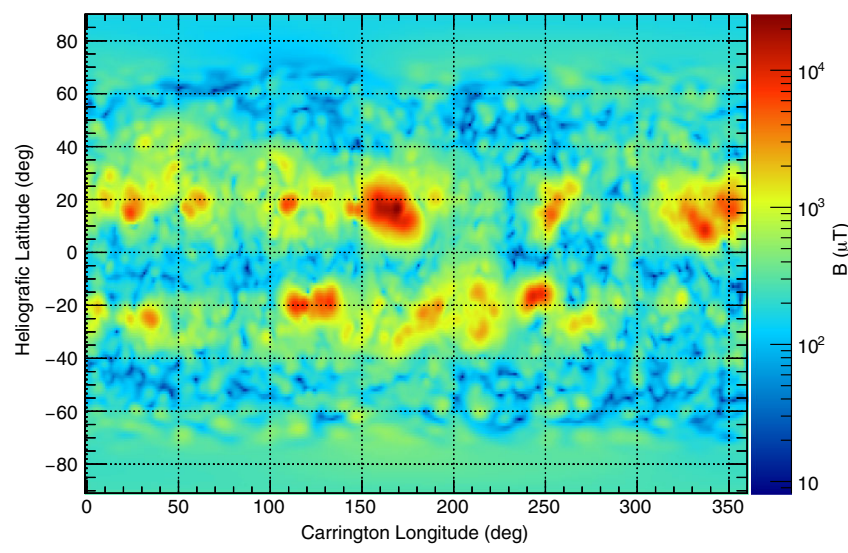

FIG. 3. Magnetic field intensity as a function of the Carrington longitude and latitude angles at $r=R_{\odot}$ for the CR 2111 .

In the present work, we assume that the magnetic field inside the Sun is always equal to that at $r=R_{\odot}$. The intensity of the magnetic field on the solar surface is shown in Fig. 3 for the CR 2111, covering the period from June 5, 2011, at 17:00 to July 3, 2011, at 0:00. We point out that in small regions of the solar surface the field intensity can even exceed $10 \mathrm{G}$.

\section{Interplanetary magnetic field}

The interplanetary magnetic field (IMF) affects the propagation of cosmic rays in the Solar System. In our simulation, we describe the IMF using the Parker model [40] for $r>R_{S S}$. The three components of the IMF are given by

$$
\begin{aligned}
& B_{r}= \pm f B_{E}\left(\frac{R_{E}}{r}\right)^{2} \\
& B_{\theta}=0 \\
& B_{\phi}=-B_{r} \tan \xi .
\end{aligned}
$$

The angle $\xi$ is defined as

$$
\tan \xi(r, \theta)=\frac{\omega_{S}\left(r-R_{S S}\right) \sin \theta}{v_{S W}}
$$

where $\theta$ is the polar angle, $\omega_{S}=2.69 \times 10^{-6} \mathrm{rad} / \mathrm{s}$ is the angular velocity of the Sun (corresponding to a period of about 27 days), and $v_{S W}$ is the velocity of the solar wind (its typical value is $400 \mathrm{~km} / \mathrm{s}$ ). At the distance $R_{S S}$, the components $B_{\phi}$ and $B_{\theta}$ are null, to ensure continuity with the PFSS model of the inner field (see Sec. II B).

In the previous equations, the intensity of the field $B_{E}$ is given by $B_{E}=B_{0} / \sqrt{1+\tan ^{2} \xi\left(R_{E}, \pi / 2\right)}$, where $B_{0}$ is the intensity of the magnetic field at the Earth (its typical value is about $5 \mathrm{nT}$ ), and $R_{E}=1 \mathrm{AU}$ is the Sun-Earth distance. The constant $f$ is given by

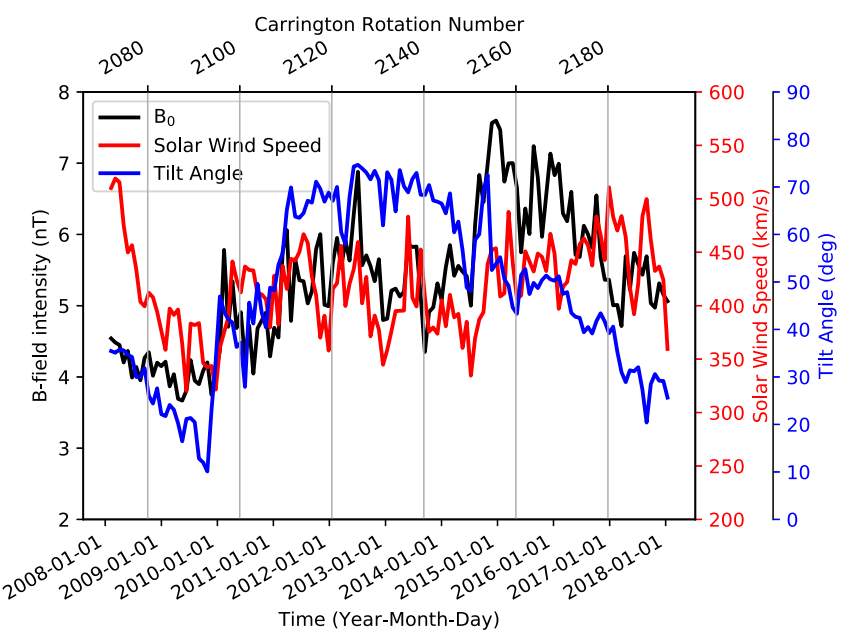

FIG. 4. Time evolution of $B_{0}$ (black line), of the solar wind speed $v_{S W}$ (red line), and of the tilt angle $\alpha$ (blue line). The upper horizontal timescale shows the CR numbers. The values of the tilt angle $\alpha$ and its polarity are taken from the Wilcox Solar Observatory public website [41]. The magnetic field at the Earth $B_{0}$ and the velocity of the solar wind $v_{S W}$ are taken from the observations of the ACE satellite extracted from the NASA/ GSFC's OMNI dataset [42,43].

$$
f=1-2 H\left(\theta-\theta^{\prime}\right),
$$

where $H$ is the Heaviside function and the angle $\theta^{\prime}$ is the polar position of the heliospheric current sheet (HCS) defined as

$$
\theta^{\prime}=\frac{\pi}{2}-\arctan \left[\tan \alpha \sin \left(\phi+\frac{\omega_{S}\left(r-R_{S S}\right)}{v_{S W}}\right)\right],
$$

where $\phi$ is the azimuth angle and we have indicated with $\alpha$ the tilt angle, i.e., the maximum latitude of the HCS; finally, the \pm sign in Eq. (1) depends on the polarity of the magnetic field.

Figure 4 shows the time evolution of the magnetic field $B_{0}$ at Earth, of the solar wind velocity $v_{S W}$, and of the tilt angle $\alpha$ averaged in each CR from 2008 to 2018. The values of the tilt angle $\alpha$ and of its polarity are taken from the Wilcox Solar Observatory public website [41], while the intensity of the magnetic field at the Earth $B_{0}$ and the velocity of the solar wind $v_{S W}$ are taken from the observations of the Advanced Composition Explorer (ACE) satellite as extracted from NASA/GSFC's OMNI data set through OMNIWeb $[42,43]$.

In our simulation, we have implemented the magnetic field configurations corresponding to a few CRs between 2011 and 2014, when the maximum of the solar cycle 24 occurred.

\section{SIMULATION RESULTS}

The yield of secondary particles produced from the $i$ th species of cosmic-ray primaries (here $i=p, e^{-}$and ${ }^{4} \mathrm{He}$ ), $Y_{s, i}\left(E_{s} \mid E_{k}\right)$, is calculated by counting the secondary 
particles which escape from the generation surface. The yield is defined as

$$
Y_{s, i}\left(E_{s} \mid E_{k}\right)=\frac{N_{s, i}\left(E_{s} \mid E_{k}\right)}{N_{i}\left(E_{k}\right) \Delta E_{s}},
$$

where $N_{i}\left(E_{k}\right)$ is the number of primaries of the $i$ th species generated with kinetic energy $E_{k}\left(E_{k}\right.$ is expressed in units of $\mathrm{GeV}$ for primary electrons and protons and of $\mathrm{GeV} / \mathrm{n}$ for primary nuclei) and $N_{s, i}\left(E_{s} \mid E_{k}\right)$ is the number of secondaries of the species $s$ with energies between $E_{s}$ and $E_{s}+$ $\Delta E_{s}$ produced by the primaries of the type $i$ with kinetic energy $E_{k}$ and escaping from the generation surface. Figure 5 shows the yields of gamma rays produced by protons (top panel), helium nuclei (middle panel), and electrons (bottom panel) as a function of the primary energy and of the gamma-ray energy.

Figure 6 shows the gamma-ray yields from primary protons for three different primary energies $(10 \mathrm{GeV}$, $100 \mathrm{GeV}$ and $1 \mathrm{TeV}$ ). At fixed primary energy, the yield roughly scales as $E_{s}^{-1}$ up to about $0.1 \mathrm{GeV}$, while above this value, it scales as $E_{s}^{-2}$. The soft component dominates the gamma-ray emission and is mainly due to the secondary production in the shower cascade for the bremsstrahlung radiation effect. This is the reason why the average gammaray energy is much lower than the energy of the parent particle. The lines at $E_{s}=511 \mathrm{keV}$, corresponding to positron annihilation, are clearly visible for all primary energies. In the case of $10 \mathrm{GeV}$ primary protons, a line at $E_{s}=2.2 \mathrm{MeV}$ is also visible, corresponding to the neutron capture process, which tends to disappear as the primary proton energy increases.

The differential intensity of secondary particles (in units of particles $\mathrm{GeV}^{-1} \mathrm{~cm}^{-2} \mathrm{sr}^{-1} \mathrm{~s}^{-1}$ ) emitted from the Sun is given by

$$
I_{s}\left(E_{s}\right)=\sum_{i} \int Y_{s, i}\left(E_{s} \mid E_{k}\right) I_{i}\left(E_{k}\right) d E_{k},
$$

where $I_{i}\left(E_{k}\right)$ is the intensity of the $i$ th species of cosmicray primaries at the Sun.

The flux of secondaries observed by a detector at Earth (in units of particles $\mathrm{GeV}^{-1} \mathrm{~cm}^{-2} \mathrm{~s}^{-1}$ ) is given by

$$
\phi_{s}\left(E_{s}\right)=\frac{\pi R_{S S}^{2}}{R_{E}^{2}} I_{s}\left(E_{s}\right) \mathcal{F}\left(E_{s}\right),
$$

where $\mathcal{F}\left(E_{s}\right)$ is the fraction of secondaries with energy $E_{s}$ which are able to reach the Earth's orbit from the Sun. In our simulation, we assume that the Earth's orbit lies on a sphere centered on the Sun with radius $r=R_{E}$. We point out here that not all secondaries emitted outward from the Sun are able to reach the Earth. Charged particles are deflected by the IMF and, depending on their energy and initial direction, can be sent back to the Sun without reaching the Earth's orbit. In addition, there are some
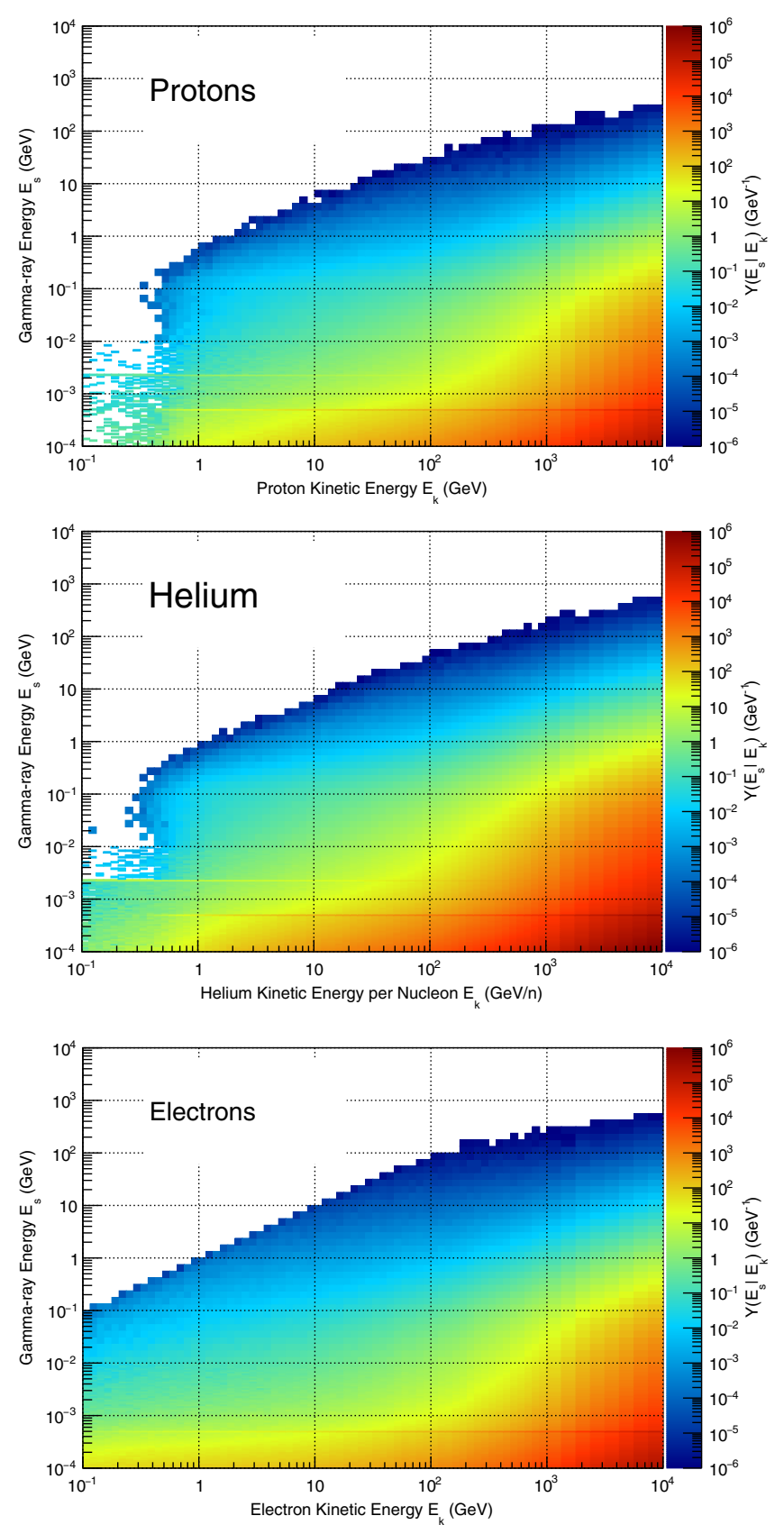

FIG. 5. Gamma-ray yields from protons (top panel), helium nuclei (middle panel), and electrons (bottom panel) as a function of the primary kinetic energy (or kinetic energy per nucleon in the case of helium primaries) (x axis) and of the gamma-ray energy (y axis). The color scale ( $\mathrm{z}$ axis) indicates the yields.

species of unstable secondaries, such as neutrons, which can decay during their journey from the Sun to the Earth. In these cases, the fraction of secondaries reaching the Earth will be $\mathcal{F}\left(E_{s}\right) \leq 1$. On the other hand, for the secondary gamma rays and neutrinos, we assume $\mathcal{F}\left(E_{s}\right)=1$. $^{2}$

\footnotetext{
${ }^{2}$ In our simulation, we neglect the possible interactions of particles with the interplanetary dust.
} 


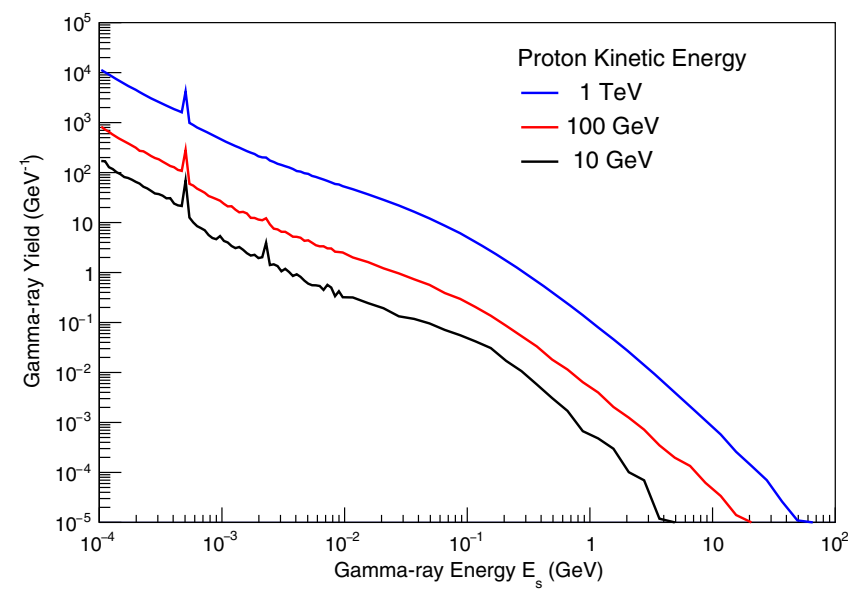

FIG. 6. Gamma-ray yields as a function of the gamma-ray energy for three different primary proton energies: $1 \mathrm{TeV}$ (blue line), $100 \mathrm{GeV}$ (red line), $10 \mathrm{GeV}$ (black line).

Cosmic rays impinging on the solar atmosphere are those which can reach the Sun from the interplanetary space. Hence, the intensities $I_{i}\left(E_{k}\right)$ of the various cosmic-ray primaries in Eq. (6) are those at the surface of the generation sphere of radius $R_{S S}$, which differ from those measured at Earth, since not all cosmic rays reaching the Earth are able to continue their journey to the Sun.

To evaluate the cosmic-ray intensities at the Sun we have used the custom code HelioProp $[44,45],{ }^{3}$ which describes the transport of cosmic rays in the Solar System. We have simulated sets of pseudoparticles injected on the surface of the generation sphere with an isotropic and uniform distribution. The pseudoparticles are followed backward in time during their propagation until they reach a sphere of radius $R_{E}$ [46-48]. Their survival probabilities are used to scale the measured intensities of cosmic rays at Earth in order to properly set the intensities $I_{i}\left(E_{k}\right)$ in the right-hand side of Eq. (6).

In our simulations, we assume that the intensity of cosmic rays measured at the Earth is the same across a sphere or radius $R_{E}=1 \mathrm{AU}$. At low energies (less than $10 \mathrm{GeV}$ ), this assumption could be not valid because of a possible dependence on the charge sign of the propagation of cosmic rays from the outer space to $1 \mathrm{AU}$ [44]. However, this effect is not expected to produce significant changes in our results, since only a small fraction of low-energy cosmic rays are able to reach the Sun.

We use the cosmic-ray intensities at Earth measured by AMS-02: the proton intensity is taken from Ref. [49], the helium intensity is taken from Refs. [50,51], and the electron ${ }^{4}$ intensity is taken from Ref. [52]. We also

\footnotetext{
${ }^{3}$ See also https://github.com/cosmicrays.

${ }^{4} \mathrm{We}$ use the total intensity of electrons and positrons, and we refer to them as electrons.
}

use the AMS-02 spectra measured for different Bartels's rotations (BRs) $[53,54] .^{5}$

Since the AMS-02 spectra are available starting from about $0.4 \mathrm{GeV} / \mathrm{n}$, we have extrapolated the data down to $0.1 \mathrm{GeV} / \mathrm{n}$ by fitting the measured intensities with a function given by [55]

$$
I\left(E_{k}\right)=a\left(E_{k}+b e^{-c \sqrt{E_{k}}}\right)^{-\alpha} .
$$

For the proton and helium, we fit the data points up to the break energy around $200 \mathrm{GeV} / \mathrm{n}$; then, for larger energies we include a smooth break with a harder spectral index, as indicated by Refs. [49,50]. In the case of electrons, we also take into account the DAMPE data [56], including a break at about $900 \mathrm{GeV}$.

Figure 7 shows the results of the fitting procedure with the experimental data points corresponding to the CR 2111, covering the period from June 5, 2011, to July 3, 2011. In Fig. 7, we also show the modulated spectra at the Sun, evaluated from those at the Earth with HelioProp.

Figure 8 shows the gamma-ray fluxes at the Earth evaluated with our simulation setup for four different CRs $(2111,2125,2138$, and 2152) spanning the period from June 2011 to June 2014, that covers the AMS-02 measurements. The calculated gamma-ray fluxes are slightly different at low energies (less than $1 \mathrm{GeV}$ ), due to the effect of the heliospheric magnetic field that affects both the cosmic-ray intensity at the Sun and the secondary yields. Finally, Fig. 9 shows the gamma-ray flux at Earth obtained by averaging the fluxes calculated in the four different CRs.

In Figs. 8 and 9, we show the total gamma-ray flux at Earth and the contributions of photons produced by the interaction of protons, helium, and electron primaries separately. The typical contributions of protons, helium, and electron primaries to the total gamma-ray fluxes are of about $74 \%, 24 \%$, and $2 \%$, respectively.

The gamma-ray flux at the Earth exhibits two sharp peaks at $511 \mathrm{keV}$ and at about $2.2 \mathrm{MeV}$, due to the positron annihilation and to the neutron capture (in the hadronic interactions), respectively. These two lines could be used as reference to calibrate the low-energy gamma-ray telescope proposed for the next decade, such as ASTROGAM $[16,17]$ and AMEGO [18]. At energies above tens of $\mathrm{GeV}$, the calculated fluxes exhibit some fluctuations that are due to the limited statistic in the simulated datasets. ${ }^{6}$

In Figs. 8 and 9, we also show the experimental results obtained with the Fermi-LAT [19] data for the disk component. The two LAT datasets correspond to a period of 1.5 years from August 2008 to January 2010, between the end of the 23rd and the beginning of the 24th solar

\footnotetext{
${ }^{5} \mathrm{~A}$ BR has a duration of exactly 27 days, close to the synodic CR of 27.2753 days. BR numbers start on February 8, 1832, while CR numbers start from November 9, 1853.

${ }^{6}$ The simulation of high-energy primaries requires a high $\mathrm{CPU}$ consumption.
} 


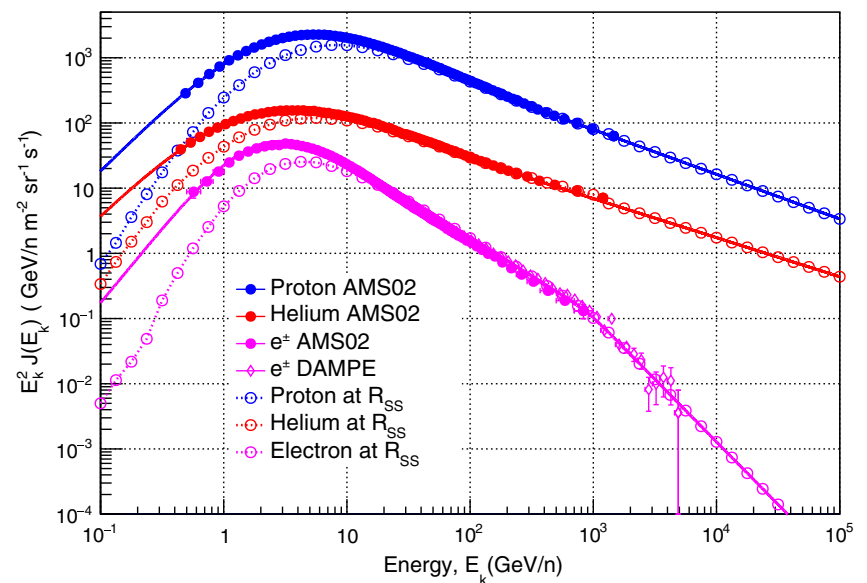

FIG. 7. Proton [49], helium [50,51], and electron intensities $[52,56]$ as functions of the kinetic energy (kinetic energy per nucleon in the case of helium). The full circles correspond to the experimental data measured by AMS-02 at Earth during CR 2111; the solid lines indicate the results of the fits; the open circles with dashed lines indicate the intensities near the Sun at $R_{S S}=2.5 \times R_{\odot}$, evaluated by scaling the intensities at Earth with the survival probabilities obtained from HelioProp [44].
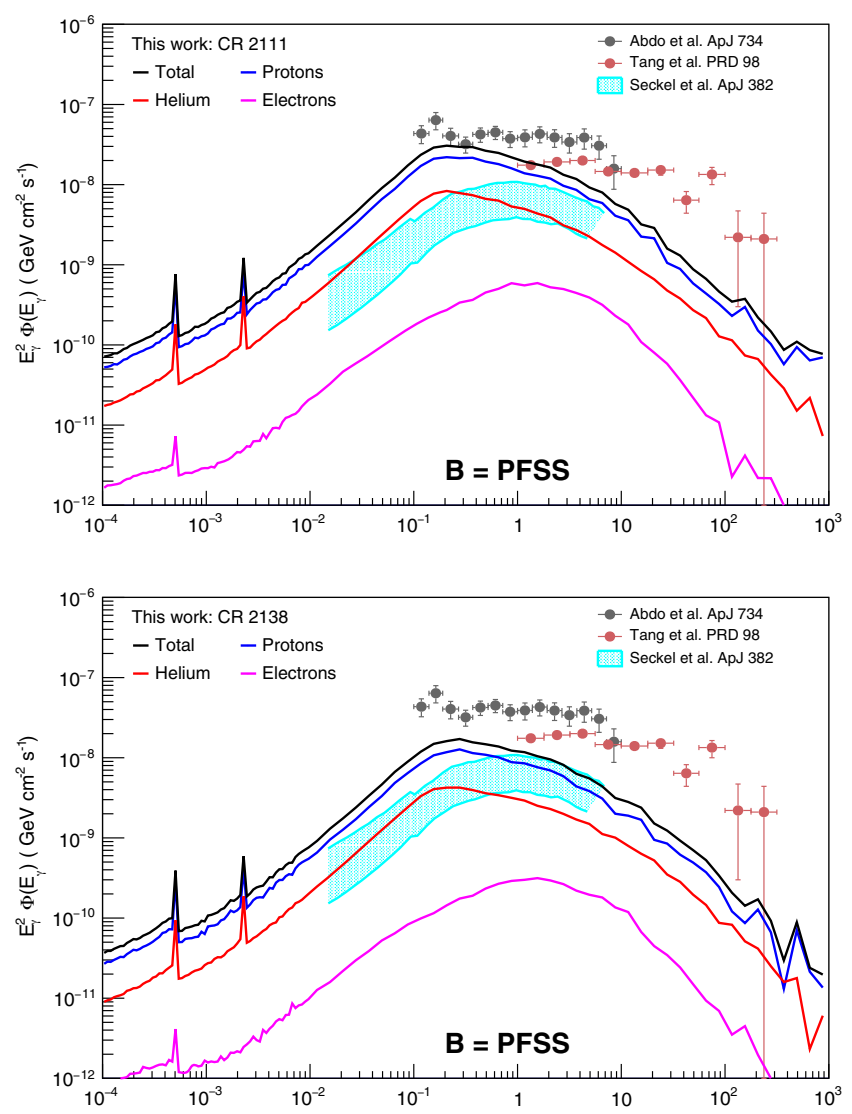

cycle [4], and to a period of 9 years from August 2008 to July 2017, spanning an almost full 11-years solar cycle [57]. We stress here that these measurements have been performed in different time windows from the one covered by our simulation. However, while our simulation predicts a peak in the spectral energy distribution of gamma rays at energies around $200 \mathrm{MeV}$, the data seem to indicate that the spectral energy distribution is almost flat up to beyond $10 \mathrm{GeV}$. This discrepancy could be due to the modeling of the inner magnetic field intensity and will be further investigated in Sec. IV. A possible cause of the discrepancy could be the modeling of the complex structure of the solar atmosphere. In addition, it could be due to the inverse Compton emission, which could produce high-energy gamma rays close to the solar surface that could be not well separated by the disk emission (we will discuss this point in Sec. V).

As we discussed in Sec. II B, the inner PFSS field maps are available on the JSOC database only starting from CR 2097 (May-June 2010), and therefore they do not cover the whole time intervals of the analyses presented in Refs. [4,57]. In addition, the AMS-02 detector on the International Space Station started its operations only in
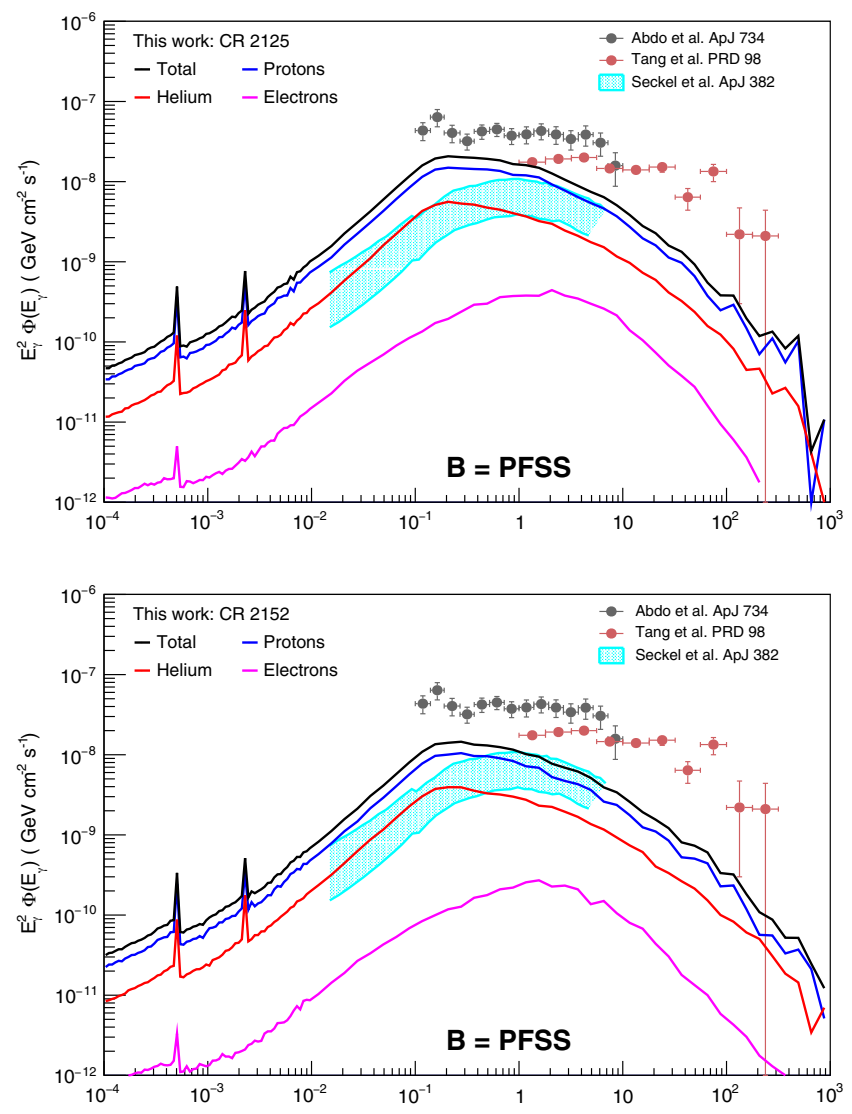

FIG. 8. Gamma-ray fluxes at the Earth for four different CRs. Top left panel: CR 2111; top right panel: CR 2125 and CR 2138 ; bottom right panel: CR 2152. Black line: total emission; blue line: gamma rays from protons; red line: gamma rays from ${ }^{4} \mathrm{He}$ nuclei; magenta line: gamma rays from electrons. Light blue shadow region: Seckel et al. model [5]; gray points: 1.5 years Fermi-LAT data [4]; dark red points: 9 years Fermi-LAT data [57]. 


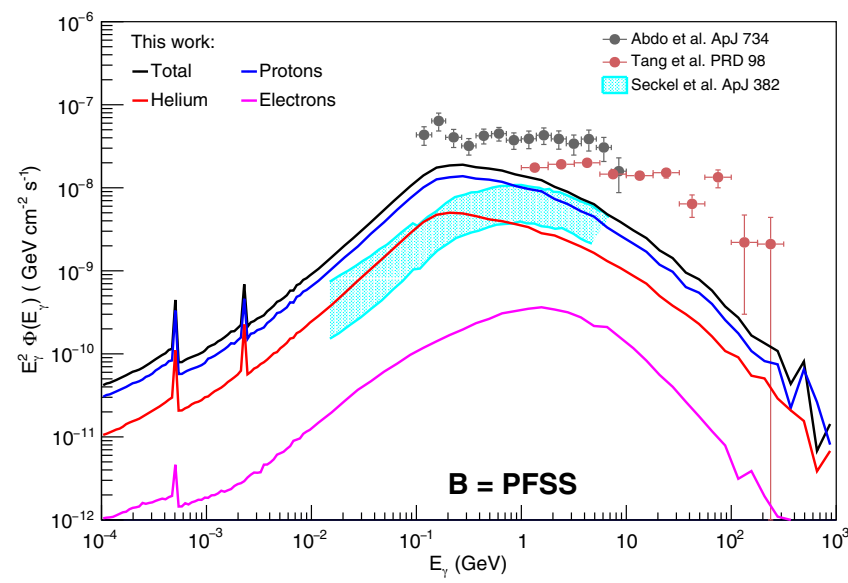

FIG. 9. Gamma-ray flux at the Earth evaluated as the average of the fluxes of the four different Carrington rotations shown in Fig. 8. Color lines and data points have the same meanings as those in Fig. 8.

May 2011, and at present, its data are available until May 2017 [53,54]. Therefore, with the simulation setup that we have implemented for this work, we are not able to make predictions on the gamma-ray flux in the period corresponding to the analysis of Ref. [4]. A detailed simulation of the whole time interval covered by the AMS-02 data would require a huge campaign, with a dedicated simulation for each CR in this period, but in any case, it would not completely overlap with the time interval analyzed in Ref. [57].

In Figs. 8 and 9, we also show the predictions of the gamma-ray flux at the Earth made by Seckel et al. [5] under their nominal assumptions, taken from Fig. 7 in Ref. [5]). The expected gamma-ray flux in each CR considered in the present work is always larger than the flux predicted in Ref. [5]. The differences can be due to the different models used for describing the solar atmosphere and the inner magnetic field and to the different approach used in the simulation. In fact, while the authors of Ref. [5] have evaluated the gamma-ray flux with a semianalytical calculation with a simplified geometry, we have implemented a full Monte Carlo simulation with the complete geometry of the Sun.

We have also cross-checked our results by backpropagating from the Sun to the Earth each particle simulated with FLUKA. Given a cosmic-ray primary at the Sun, a particle with opposite charge and with opposite direction is backpropagated from the generation sphere of radius $R_{S S}$ to the sphere of radius $R_{E}$. If this particle is able to reach the Earth, the primary particle is assigned a survival probability $P_{\text {surv }}=1$; otherwise, it is assigned $P_{\text {surv }}=0$. With this procedure, the secondary yield can be calculated as

$$
Y_{s, i}\left(E_{s} \mid E_{k}\right)=\frac{N_{s, i}\left(E_{s} \mid E_{k}, P_{\text {surv }}=1\right)}{N_{i}\left(E_{k}\right) \Delta E_{s}},
$$

TABLE I. Gamma-ray fluxes at Earth above $100 \mathrm{MeV}, 1 \mathrm{GeV}$, and $10 \mathrm{GeV}$, respectively, in four different Carrington rotations.

\begin{tabular}{lccc}
\hline \hline $\mathrm{CR}$ & $\begin{array}{c}\Phi_{\gamma}(>100 \mathrm{MeV}) \\
\times 10^{-7} \mathrm{~cm}^{-2} \mathrm{~s}^{-1}\end{array}$ & $\begin{array}{c}\Phi_{\gamma}(>1 \mathrm{GeV}) \\
\times 10^{-8} \mathrm{~cm}^{-2} \mathrm{~s}^{-1}\end{array}$ & $\begin{array}{c}\Phi_{\gamma}(>10 \mathrm{GeV}) \\
\times 10^{-9} \mathrm{~cm}^{-2} \mathrm{~s}^{-1}\end{array}$ \\
\hline 2111 & $2.59 \pm 0.02$ & $1.42 \pm 0.02$ & $2.61 \pm 0.10$ \\
2125 & $1.79 \pm 0.01$ & $1.16 \pm 0.02$ & $2.19 \pm 0.08$ \\
2138 & $1.38 \pm 0.01$ & $0.84 \pm 0.02$ & $1.66 \pm 0.06$ \\
2152 & $1.23 \pm 0.01$ & $0.74 \pm 0.02$ & $1.51 \pm 0.05$ \\
\hline \hline
\end{tabular}

where $N_{i}\left(E_{k}\right)$ is the number of primaries of the $i$ th species generated with kinetic energy $E_{k}$ and $N_{s, i}\left(E_{s} \mid E_{k}\right.$, $\left.P_{\text {surv }}=1\right)$ is the number of secondaries of the $i$ th species with energies between $E_{s}$ and $E_{s}+\Delta E_{s}$ produced by the primaries of the type $i$ with kinetic energy $E_{k}$ and $P_{\text {surv }}=1$. In this way, the secondary spectra at the Earth can be calculated inserting in the right-hand side of Eq. (6) the proton, helium, and electron intensities measured at the Earth. Using this procedure, we find the same results as when we evaluate the intensities of primary CRs at the Sun with HelioProp.

In Table I, we show the integral fluxes of gamma rays at Earth above $100 \mathrm{MeV}, 1 \mathrm{GeV}$, and $10 \mathrm{GeV}$, respectively, for the four CRs considered in this section. The integral flux decreases with increasing CR number, as the Sun approaches to its maximum activity.

The secondary productions at high energies occur close the solar surface, where the secondary are emitted in a low-density medium in the forward direction with respect to the high-energy primary particles. However, the combination of the solar magnetic field with the solar atmosphere density profile can affect the emission, even at high energies. Figure 10 shows the gamma-ray flux seen at the Earth as a function of the angle of sight for two different energy bins, i.e., [0.1, 0.133] and [1, 1.33] GeV. We also show the corresponding spatial emission maps centered on the Sun and built with the HEALPix pixelization [58]. ${ }^{7}$ The emission is mainly located nearby the solar surface, and it becomes much narrow at higher energies.

Figure 11 shows the intensity of different species of secondaries (muon neutrinos and antineutrinos, electron neutrinos and antineutrinos, neutrons, electrons, and positrons) produced by the interactions of cosmic rays with the Sun, evaluated on the generation sphere. The values of the intensities are obtained by averaging the results in the four CRs mentioned above. To calculate the fluxes at the Earth, the decays of unstable particles during their journey from the Sun to the Earth should be taken into account, in addition to the propagation of charged particles in the IMF. We also remark here that in the calculation of the neutrino and antineutrino fluxes we did not include their interactions in the Sun (their absorption is negligible below $10 \mathrm{GeV}$ )

\footnotetext{
${ }^{7}$ HEALPix website: currently http://healpix.sourceforge.net or https://healpix.sourceforge.io.
} 

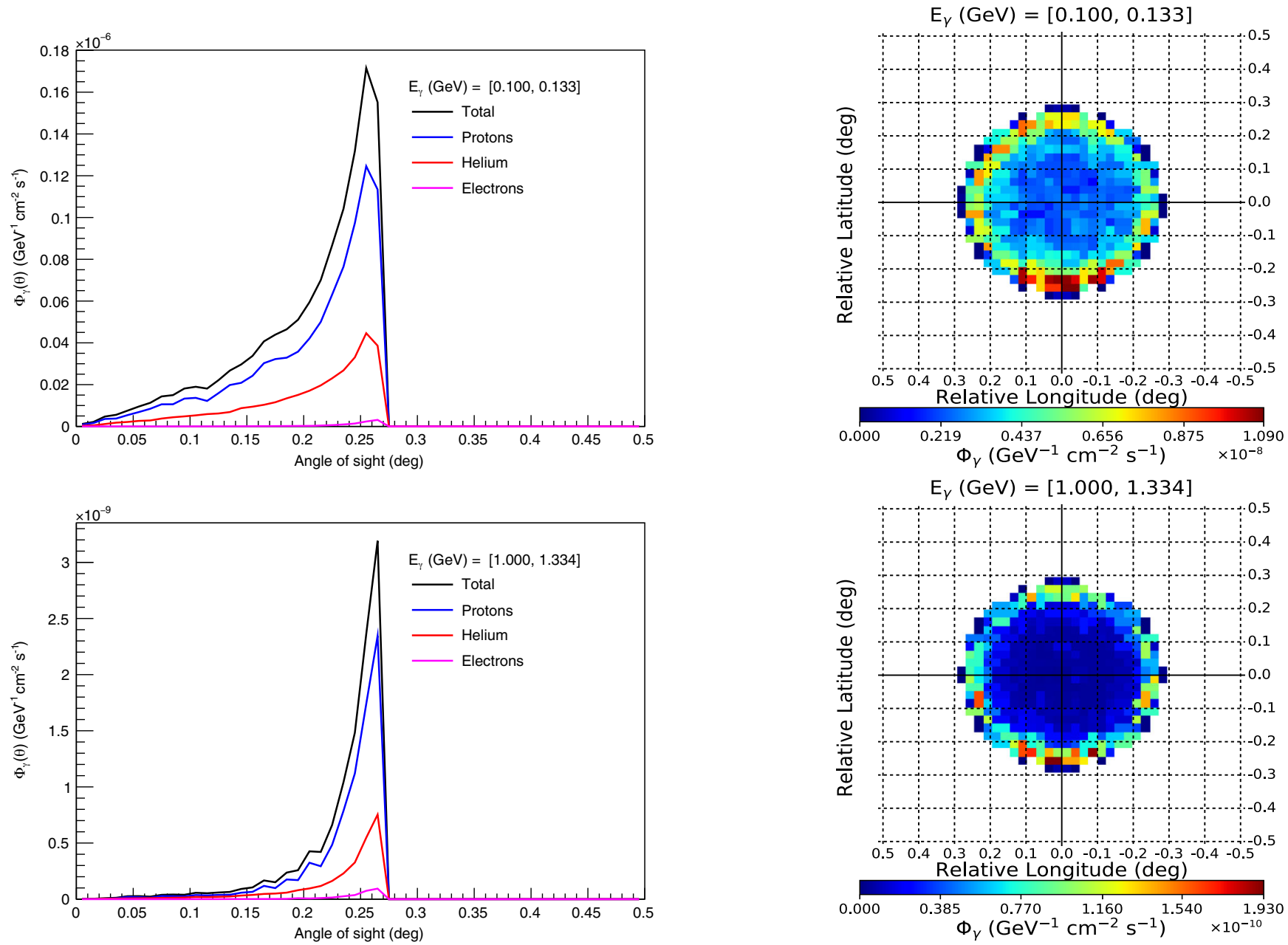

FIG. 10. Left panels: gamma-ray fluxes at the Earth as a function of the angle of sight. Right panels: spatial map of the solar gammaray emission built with the HEALPix pixelization with $N_{\text {side }}=2048$. Two different energy bins are considered: [0.1, 0.133$]$ (top row) and $[1,1.33] \mathrm{GeV}$ (bottom row).

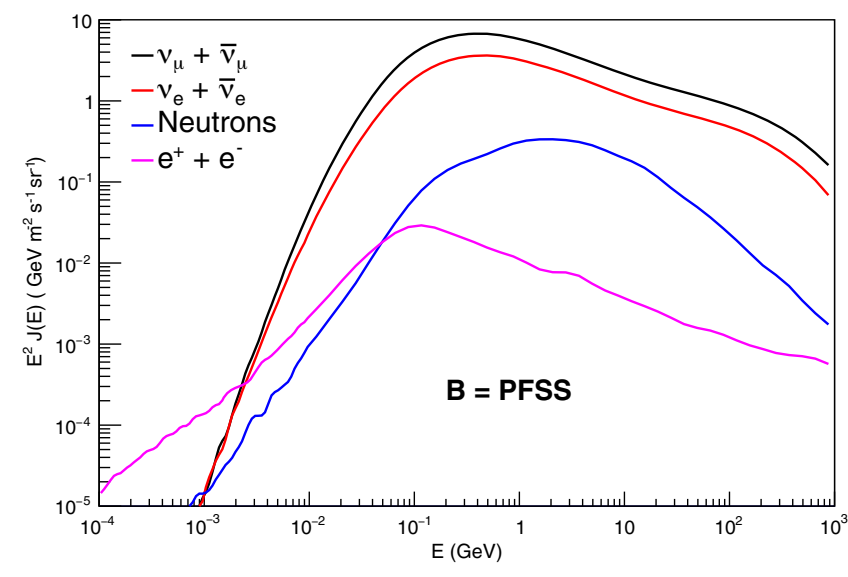

FIG. 11. Average intensity at the Sun of $\nu_{\mu}+\bar{\nu}_{\mu}$ (black line), $\nu_{e}+\bar{\nu}_{e}$ (red line), neutrons (blue line), $e^{+}+e^{-}$(magenta line) for CRs 2111, 2125, 2138, and 2152. and their possible oscillations [9]. The expected neutrino intensity is similar to that calculated in Ref. [9], and above $100 \mathrm{GeV}$, it is higher than the intensity of neutrinos produced in cosmic-ray showers in the Earth's atmosphere (see, for example, Ref. [59]).

\section{EFFECT OF THE MAGNETIC FIELD ON THE SECONDARY YIELDS}

The secondary emissivity of the Sun is strongly dependent on the intensity of the magnetic field close to the solar surface. To study this effect, we have implemented in our simulation three additional magnetic field configurations for the CR 2111:

(1) $B=0$, i.e., we switch the magnetic field off;

(2) $0.1 \times$ PFSS, i.e., we reduce the original PFSS magnetic field intensity of a factor 10 ;

(3) enhanced $B$ field configuration near the $\operatorname{Sun}\left(r / R_{\odot}<\right.$ 1.01) following the BIFROST model [60-62], i.e., 

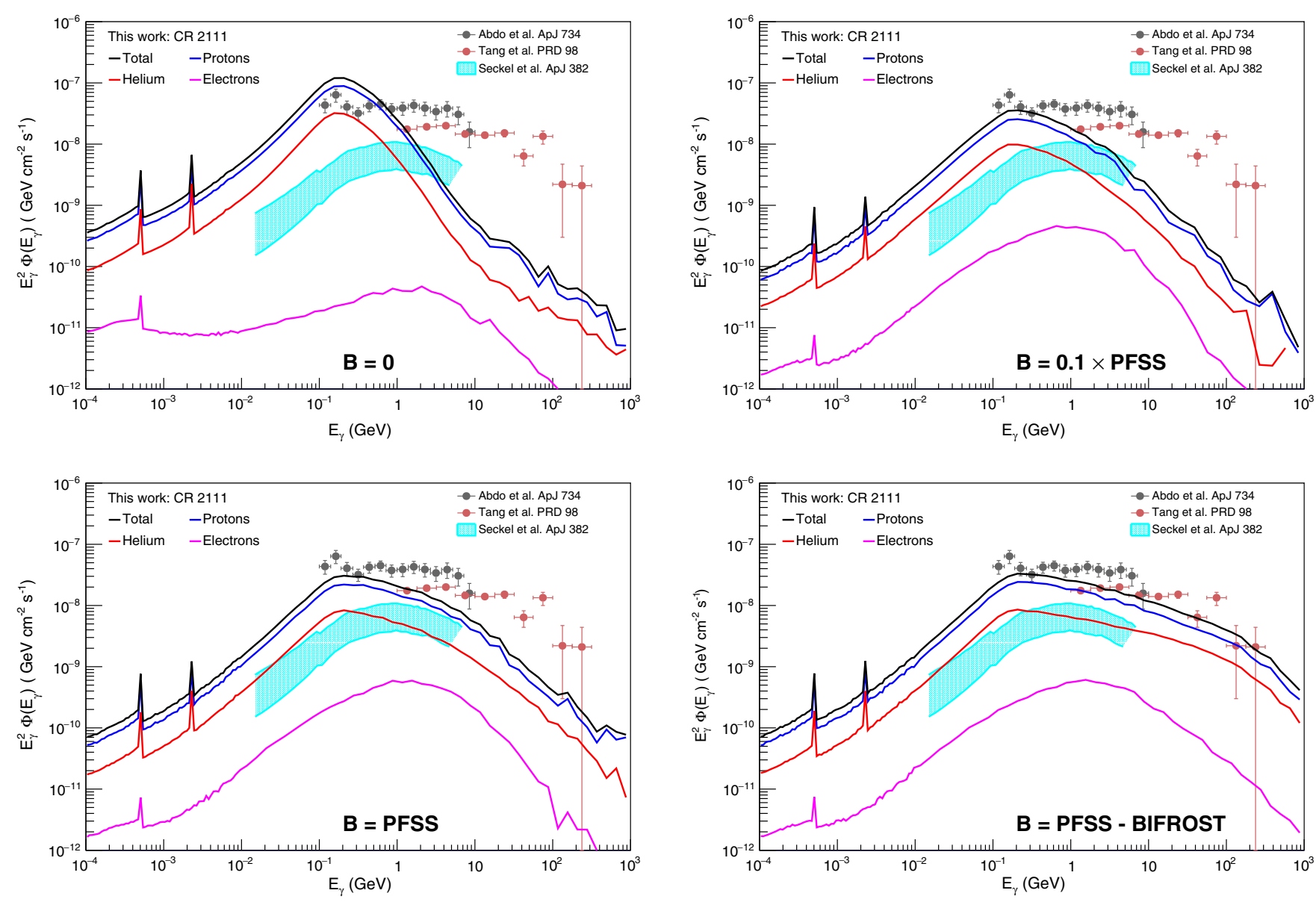

FIG. 12. Gamma-ray fluxes at the Earth for the four different magnetic field configurations. Top left panel: no $B$ field; top right panel: $B$ reduced by a factor 10 with respect to the nominal PFSS configuration; bottom left panel: PFSS configuration; bottom right panel: enhanced magnetic field according to the BIFROST model. Color lines and data points have the same meanings as in Fig. 8.

we increase the original PFSS maps near the Sun to follow the BIFROST profile. ${ }^{8}$

Figure 12 shows the gamma-ray fluxes at the Earth with the four different configurations of the inner magnetic field, i.e., the nominal model and the three alternative models illustrated above.

The gamma-ray flux without magnetic field is significantly enhanced at low energies with respect to the flux in presence of magnetic field, while for gamma-ray energies above $10 \mathrm{GeV}$, the flux increases as the magnetic field increases. If the solar magnetic field is suppressed, lowenergy cosmic rays can reach the Sun surface, inducing a shower which produces secondary particles in the outer space. The presence of a solar magnetic field reduces the probability that low-energy cosmic rays can reach the Sun but increases the probability of interaction for high-energy cosmic rays, since they move along curved trajectories in a strong and nonuniform magnetic field and their path length

\footnotetext{
${ }^{8}$ The BIFROST simulation is available for a limited region of the Sun. The enhancement factor is about 25 at the solar surface.
}

increases as the magnetic field increases. This effect is very visible when comparing the gamma-ray fluxes with $B=0$ (top left panel in Fig. 12) with the one with enhanced $B$ field configuration (bottom right panel in Fig. 12)

\section{DISCUSSION AND CONCLUSIONS}

We have implemented a full simulation with the FLUKA code to calculate the yields of secondary particles produced by the interactions of primary cosmic rays with the solar atmosphere. Our simulation includes the current state-of-art models and data available to describe the solar atmosphere, the magnetic field nearby the Sun, and the interplanetary space.

The FLUKA toolkit provides a detailed simulation of hadronic and electromagnetic interactions in the matter in a wide energy range, with complex geometries and even in the presence of magnetic fields. The geometry used in the present work is quite flexible, and it can be used for any other configuration.

The solar atmosphere and its chemical composition have been taken from the SSM gs98 and from model S, with some extrapolation in the chromosphere region. However, 


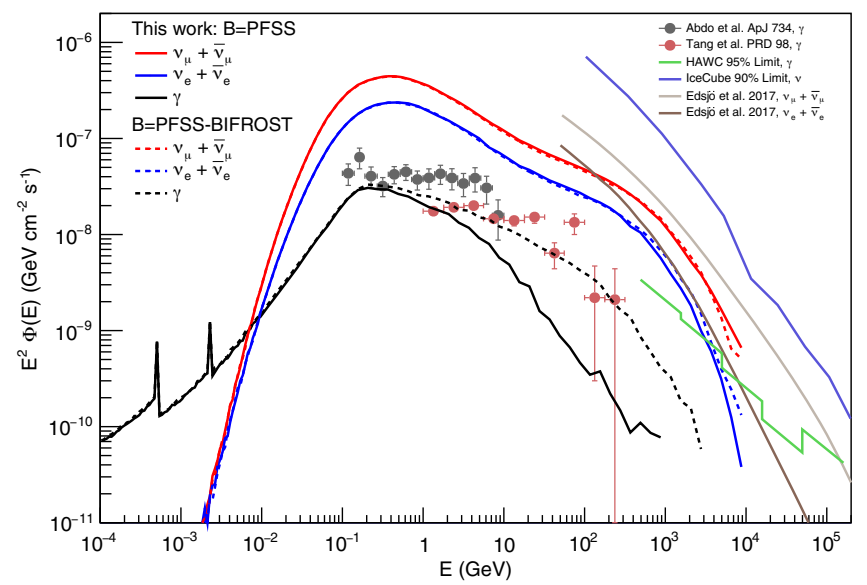

FIG. 13. Gamma-ray and neutrino fluxes at the Earth calculated for the Carrington rotation 2111 with two models for the Sun magnetic field: PFSS (continuous line) and enhanced model according to the BIFROST prediction (dashed line). Black line: gamma-ray; red line: $\nu_{\mu}+\bar{\nu}_{\mu}$; blue line: $\nu_{e}+\bar{\nu}_{e}$. The prediction of neutrinos by Ref. [9] is also shown. In addition, we include results from gamma-ray observations from Fermi-LAT [4,57], gamma-ray HAWC's 95\% limit [65], and neutrinos IceCube's 90\% limit [66].

the average density should drop below $10^{-13} \mathrm{~g} / \mathrm{cm}^{3}$ at an altitude of about $1400 \mathrm{~km}$ from the solar surface, where the interaction probability should be negligible. We have also used the model ags09, ${ }^{9}$ and we found very similar results.

The magnetic field adopted nearby the Sun is the one predicted by the PFSS model by using the synoptic map from HMI 720s line-of-sight magnetograms collected over 27-day solar rotations in a high-resolution Carrington coordinate grid. We have studied the effect of the magnetic field on the secondary yields by changing the original values of the PFSS maps. Indeed, we found that the yields are strongly affected by the intensity of the magnetic field, even in the high-energy region of the emission in the outer space. Recent developments of numerical solutions of a magneto-hydrodynamical model together with the current observation of the Parker Solar Probe [63,64] could provide new insights to get a realistic description of the plasma dynamics and of the magnetic field nearby the Sun.

The calculated solar gamma-ray flux at the Earth has been compared with the Fermi-LAT data on the disk emission in different time windows with respect to those used in the present simulations. The detected gamma-ray emission from the solar disk above $1 \mathrm{GeV}$ shows a harder spectrum (approximately $E_{\gamma}^{-2.2}$ ) than the cosmic-ray spectrum (approximately $E_{p, \mathrm{He}}^{-2.7}$ ). This behavior would require a high-intensity magnetic field configuration nearby the Sun, up to a factor 20 larger than the field predicted by the PFSS model used in the present simulation.

\footnotetext{
${ }^{9}$ http://www.ice.csic.es/personal/aldos/Solar_Data_files/ struct_b16_agss09.dat.
}

In Fig. 13, we show the predictions of our simulation about the fluxes at Earth of gamma rays and neutrinos in the CR 2111. The simulations have been performed with the standard PFSS solar magnetic field and with the enhanced one according to the BIFROST profile. The gamma-ray production is higher in the case of the more intense magnetic field, while the effect of the magnetic field on the neutrino production seems negligible. This could be a signature that the interactions in high magnetic field occur in the higher layers of the low-density solar atmosphere, resulting in an enhanced high-energy gamma-ray emission. Anyway, above $100 \mathrm{GeV}$ the predicted gamma-ray flux is below the HAWC's limit [65], even in the case of the enhanced solar magnetic field. The predicted neutrino flux is lower than the calculation by Ref. [9], in particular below $1 \mathrm{TeV}$, due to the effect of the nearby solar magnetic field and is well below IceCube's 90\% limit [66].

The magnetic field should also affect the inverse Compton gamma-ray emission close to the Sun, since the electrons should move along curved trajectories, whose lengths determine the interaction probability with the intense optical photon field. In this way, the inverse Compton emission should also be peaked close to the solar surface and might be not well separated by the disk emission due to the interaction of cosmic rays with the solar atmosphere. To get a complete picture of the solar gamma-ray emission, the inverse Compton scattering needs to be calculated in the presence of strong and irregular magnetic field. The current model relies on a simple calculation, in which it is assumed that electrons move along straight line [6-8]. The simulation of the inverse Compton scattering in the presence of magnetic field is beyond the scope of the current paper, and it deserves a dedicated work.

\section{ACKNOWLEDGMENTS}

We acknowledge the FLUKA Collaboration for providing and supporting the code. We acknowledge use of Joint Science Operations Center data. We acknowledge use of NASA/GSFC's Space Physics Data Facility's OMNIWeb (or CDAWeb or ftp) service and OMNI data. We acknowledge use of simulation results provided by the Community Coordinated Modeling Center at Goddard Space Flight Center through their public Runs on Request system (http:// ccmc.gsfc.nasa.gov). We acknowledge use of simulation results provided by the Hinode Science Data Centre Europe (http://sdc.uio.no/search/simulations). Some of the results in this paper have been derived using the HEALPix [K. M. Górski et al., 2005, Astrophys. J. 622, 759 (2005)] package. We thank J. Todd Hoeksema for his useful discussion on the PFSS model. This work has been realized using the RECAS computing infrastructure in Bari (https:// www.recas-bari.it/index.php/en/). A particular acknowledgment goes to G. Donvito and A. Italiano for their valuable support (Instituto Nazionale di Fisica Nucleare). In this work, we used custom software based on Fortran, C ++ , Python languages and the ROOT toolkit [67]. 
[1] A. A. Abdo et al., Astrophys. J. 758, 140 (2012).

[2] M. Ackermann et al. (Fermi-LAT Collaboration), Phys. Rev. D 93, 082001 (2016).

[3] A. A. Abdo et al. (Fermi-LAT Collaboration), Phys. Rev. D 80, 122004 (2009).

[4] A. A. Abdo et al. (Fermi-LAT Collaboration), Astrophys. J. 734, 116 (2011).

[5] D. Seckel, T. Stanev, and T. K. Gaisser, Astrophys. J. 382, 652 (1991).

[6] E. Orlando and A. W. Strong, Astron. Astrophys. 480, 847 (2008).

[7] E. Orlando and A. Strong, Astrophys. Space Sci. 309, 359 (2007).

[8] I. V. Moskalenko, T. A. Porter, and S. W. Digel, Astrophys. J. 652, L65 (2006); 664, L143 (2007).

[9] J. Edsjö, J. Elevant, R. Enberg, and C. Niblaeus, J. Cosmol. Astropart. Phys. 06 (2017) 033.

[10] K. C. Y. Ng, J. F. Beacom, A. H. G. Peter, and C. Rott, Phys. Rev. D 96, 103006 (2017).

[11] B. Zhou, K. C. Y. Ng, J. F. Beacom, and A. H. G. Peter, Phys. Rev. D 96, 023015 (2017).

[12] M. Masip, Astropart. Phys. 97, 63 (2018).

[13] A. Cuoco, P. De La Torre Luque, F. Gargano, M. Gustafsson, F. Loparco, M. N. Mazziotta, and D. Serini, Phys. Rev. D 101, 022002 (2020).

[14] C. A. Argüelles, G. de Wasseige, A. Fedynitch, and B. J. P. Jones, J. Cosmol. Astropart. Phys. 07 (2017) 024.

[15] G. Ingelman and M. Thunman, Phys. Rev. D 54, 4385 (1996).

[16] A. De Angelis et al. (e-ASTROGAM Collaboration), Exper. Astron. 44, 25 (2017).

[17] M. Tavani et al. (e-ASTROGAM Collaboration), JHEAp 19, 1 (2018).

[18] J. McEnery et al. (AMEGO Collaboration), arXiv:1907.07558.

[19] W. B. Atwood et al. (Fermi-LAT Collaboration), Astrophys. J. 697, 1071 (2009).

[20] M. N. Mazziotta, F. Cerutti, A. Ferrari, D. Gaggero, F. Loparco, and P. R. Sala, Astropart. Phys. 81, 21 (2016).

[21] A. Ferrari, P. R. Sala, A. Fassò, and J. Ranft, FLUKA: A multi-particle transport code, https://doi.org/10.2172/877507, 2005.

[22] T. Böhlen, F. Cerutti, M. Chin, A. Fassò, A. Ferrari, P. Ortega, A. Mairani, P. Sala, G. Smirnov, and V. Vlachoudis, Nucl. Data Sheets 120, 211 (2014).

[23] G. Battistoni, T. Böhlen, F. Cerutti, P. W. Chin, L. S. Esposito, A. Fassò, A. Ferrari, A. Lechner, A. Empl, A. Mairani, A. Mereghetti, P. G. Ortega, J. Ranft, S. Roesler, P. R. Sala, V. Vlachoudis, and G. Smirnov, Ann. Nucl. Energy 82, 10 (2015), joint International Conference on Supercomputing in Nuclear Applications and Monte Carlo 2013, SNA + MC 2013. Pluri- and Trans-disciplinarity, Towards New Modeling and Numerical Simulation Paradigms.

[24] S. Roesler, R. Engel, and J. Ranft, in Advanced Monte Carlo for Radiation Physics, Particle Transport Simulation and Applications, edited by A. Kling, F. J. C. Baräo, M. Nakagawa, L. Távora, and P. Vaz (Springer, Berlin, 2001), pp. 1033-1038, https://doi.org/10.1007/978-3-642-18211$2 \_166$.
[25] A. Fassò, A. Ferrari, P. R. Sala, and J. Ranft, in Advanced Monte Carlo for Radiation Physics, Particle Transport Simulation and Applications. Proceedings, Conference, MC2000, Lisbon, Portugal, 2000 (Springer-Verlag, Berlin, Lisbon, 2000), pp. 955-960, https://doi.org/10.1007/978-3642-18211-2_153.

[26] G. Battistoni, F. Cerutti, R. Engel, A. Fassò, A. Ferrari, E. Gadioli, M. Garzelli, J. Ranft, S. Rösler, and P. Sala, in 12th International Conference on Nuclear Reaction Mechanisms, Villa Monastero, Varenna, Italy (CERN, Geneva, 2006), pp. 483-495, http://cds.cern.ch/record/1233497/.

[27] N. Vinyoles, A. M. Serenelli, F. L. Villante, S. Basu, J. Bergström, M. C. Gonzalez-Garcia, M. Maltoni, C. Peña-Garay, and N. Song, Astrophys. J. 835, 202 (2017).

[28] J. Christensen-Dalsgaard et al., Science 272, 1286 (1996).

[29] K. H. Schatten, J. M. Wilcox, and N. F. Ness, Sol. Phys. 6, 442 (1969).

[30] K. Hakamada, Sol. Phys. 159, 89 (1995).

[31] P. H. Scherrer, R. S. Bogart, R. I. Bush, J. T. Hoeksema, A. G. Kosovichev, J. Schou, W. Rosenberg, L. Springer, T. D. Tarbell, A. Title, C. J. Wolfson, and I. Zayer, and The MDI Engineering Team, Sol. Phys. 162, 129 (1995).

[32] http://jsoc.stanford.edu/ajax/lookdata.html?ds=hmi.pfss_ synop.

[33] V.S. Titov, Z. Mikic, J. A. Linker, and R. Lionello, Astrophys. J. 675, 1614 (2008).

[34] S. K. Antiochos, Z. Mikić, V. S. Titov, R. Lionello, and J. A. Linker, Astrophys. J. 731, 112 (2011).

[35] X. Sun, Y. Liu, J. T. Hoeksema, K. Hayashi, and X. Zhao, Sol. Phys. 270, 9 (2011).

[36] http://hmi.stanford.edu/.

[37] https://sdo.gsfc.nasa.gov/.

[38] http://soi.stanford.edu/.

[39] https://sohowww.nascom.nasa.gov/.

[40] E. N. Parker, Astrophys. J. 128, 664 (1958).

[41] http://wso.stanford.edu/Tilts.html.

[42] https://omniweb.sci.gsfc.nasa.gov/ftpbrowser/ace_merge .html.

[43] J. H. King and N. E. Papitashvili, J. Geophys. Res.: Space Phys. 110, A02104 (2005).

[44] L. Maccione, Phys. Rev. Lett. 110, 081101 (2013).

[45] A. Vittino, C. Evoli, and D. Gaggero, Proc. Sci., ICRC2017 (2018) 024 [arXiv:1707.09003].

[46] R. D. Strauss, M. S. Potgieter, I. Büsching, and A. Kopp, Astrophys. J. 735, 83 (2011).

[47] R. D. Strauss, M. S. Potgieter, I. Büsching, and A. Kopp, Astrophys. Space Sci. 339, 223 (2012).

[48] K. Alanko-Huotari, I. G. Usoskin, K. Mursula, and G. A. Kovaltsov, J. Geophys. Res.: Space Phys. 112, A08101 (2007).

[49] M. Aguilar et al. (AMS Collaboration), Phys. Rev. Lett. 114, 171103 (2015).

[50] M. Aguilar et al. (AMS Collaboration), Phys. Rev. Lett. 115, 211101 (2015).

[51] M. Aguilar et al. (AMS Collaboration), Phys. Rev. Lett. 119, 251101 (2017).

[52] M. Aguilar et al. (AMS Collaboration), Phys. Rev. Lett. 113, 221102 (2014). 
[53] M. Aguilar et al. (AMS Collaboration), Phys. Rev. Lett. 121, 051101 (2018).

[54] M. Aguilar et al. (AMS Collaboration), Phys. Rev. Lett. 121, 051102 (2018).

[55] T. K. Gaisser, T. Stanev, M. Honda, and P. Lipari, in 27th International Cosmic Ray Conference (ICRC 2001) Hamburg, Germany, 2001 (CERN, Geneva, 2001), pp. 1643-1646, http://icrc2001.uni-wuppertal.de/ICRC2001/ papers/ici6694_p.pdf.

[56] G. Ambrosi et al. (DAMPE Collaboration), Nature (London) 552, 63 (2017).

[57] Q.-W. Tang, K. C. Y. Ng, T. Linden, B. Zhou, J. F. Beacom, and A. H. G. Peter, Phys. Rev. D 98, 063019 (2018).

[58] K. M. Gorski, E. Hivon, A. J. Banday, B. D. Wandelt, F. K. Hansen, M. Reinecke, and M. Bartelmann, Astrophys. J. 622, 759 (2005).

[59] J. I. Illana, P. Lipari, M. Masip, and D. Meloni, Astropart. Phys. 34, 663 (2011).

[60] M. Carlsson, V. H. Hansteen, B. V. Gudiksen, J. Leenaarts, and B. De Pontieu, Astron. Astrophys. 585, A4 (2016).
[61] B. V. Gudiksen, M. Carlsson, V. H. Hansteen, W. Hayek, J. Leenaarts, and J. Martinez-Sykora, Astron. Astrophys. 531, A154 (2011).

[62] http://sdc.uio.no/search/simulations?sim=en024048_hion.

[63] N. J. Fox, M. C. Velli, S. D. Bale, R. Decker, A. Driesman, R. A. Howard, J. C. Kasper, J. Kinnison, M. Kusterer, D. Lario, M. K. Lockwood, D. J. McComas, N. E. Raouafi, and A. Szabo, Space Sci. Rev. 204, 7 (2016).

[64] P. Riley, C. Downs, J. A. Linker, Z. Mikic, R. Lionello, and R. M. Caplan, Astrophys. J. 874, L15 (2019).

[65] A. Albert et al. (HAWC Collaboration), Phys. Rev. D 98, 123011 (2018).

[66] M. G. Aartsen et al. (IceCube Collaboration), arXiv:1912 .13135 .

[67] R. Brun and F. Rademakers, Nucl. Instrum. Methods A 389, 81 (1997).

Correction: The top left panel of the previously published Figure 12 contained a plotting error and has been replaced. 